\title{
Spatial Spread of Tuberculosis through Neighborhoods Segregated by Socioeconomic Position: A Stochastic Automata Model
}

\author{
David Rehkopf, ${ }^{1}$ Alice Furumoto-Dawson, ${ }^{2}$ \\ Anthony Kiszewski, ${ }^{3}$ and Tamara Awerbuch-Friedlander ${ }^{4}$ \\ ${ }^{1}$ Department of Medicine, School of Medicine, Stanford University, Medical School Office Building, 251 Campus Drive, \\ Room X3c46, MC5411, Stanford, CA 94305, USA \\ ${ }^{2}$ Program on the Global Environment, The University of Chicago, 5828 S. University Avenue, Pick 101, Chicago, IL 60637, USA \\ ${ }^{3}$ Department of Natural and Applied Sciences, Bentley University, 175 Forest Street, Waltham, MA 02452, USA \\ ${ }^{4}$ Department of Global Health and Population, Harvard School of Public Health, 665 Huntington Avenue, Room 1219, \\ Boston, MA 02115, USA
}

Correspondence should be addressed to Tamara Awerbuch-Friedlander; tamara@hsph.harvard.edu

Received 25 March 2015; Revised 20 June 2015; Accepted 25 June 2015

Academic Editor: Aleksei A. Koronovskii

Copyright (C) 2015 David Rehkopf et al. This is an open access article distributed under the Creative Commons Attribution License, which permits unrestricted use, distribution, and reproduction in any medium, provided the original work is properly cited.

\begin{abstract}
Transmission of the agent of tuberculosis, Mycobacterium tuberculosis, is dependent on social context. A discrete spatial model representing neighborhoods segregated by levels of crowding and immunocompetence is constructed and used to evaluate prevention strategies, based on a number of assumptions about the spatial dynamics of tuberculosis. A cellular automata model is used to (a) construct neighborhoods of different densities, (b) model stochastically local interactions among individuals, and (c) model the spread of tuberculosis within and across neighborhoods over time. Since infected people may become progressively sick but also heal through treatment, the transition among stages was modeled with transition probabilities. A moderate level of successful treatment (40\%) dramatically reduced the number of infections across all neighborhoods. Increasing the treatment in neighborhoods of a lower socioeconomic level from $40 \%$ to $90 \%$ results in an additional decrease of approximately $25 \%$ in the number of infected individuals overall. In conclusion, we find that a combination of a moderate level of successful treatment across all areas with more focused treatment efforts in lower socioeconomic areas resulted in the least number of infections over time.
\end{abstract}

\section{Introduction and Background}

Cellular automata (if a deterministic model) and stochastic automata (if implemented with varying probabilities of transmission) are methods that allow us to study the dynamics of the population at large, based on local interactions among neighbors, as shown in models of physical systems [1]. Cellular automata consist of a lattice of cells and proceed through discrete time steps where each cell is characterized by a state and where its future states are determined stochastically in relation to a finite number of neighboring cells. Its applicability in biology was validated by implementing it in a study of a homogeneous population where individuals interact locally, resulting in the same dynamics as the one obtained with difference equations [2]. Thus it is possible with this method to follow the spread of disease in a population at large by looking at interactions among individuals [3-8]. The method is being applied here, to study the spread of tuberculosis and identify sites for treatment.

Tuberculosis is both an ancient, familiar infectious disease (the White Plague, "consumption") and a dangerous, resurgent threat to the lives and health of millions worldwide $[9,10]$. Rising rates of comorbidities that undermine the immunocompetence of populations; the migration of populations dislocated by conflict, market failures, and economic transitions [11-13]; incarceration, homelessness, and crowding in inadequate housing for disadvantaged groups living in high income inequity countries [14]; defunding of barely adequate screening and treatment programs; the emergence of drug-resistant strains out of inadequately treated prison, 
HIV positive, and homeless populations all contribute to sustained levels of tuberculosis disease (TB) in the USA and worldwide. Combinations of conditions such as these have produced alarming spikes in active $\mathrm{TB}$ case rates and $\mathrm{TB}$ mortality across the globe. One such spike occurred in the USA in the late 1980s, fueled by economic downturn, lagging public health control efforts, and the rising incidence of what was then a relatively new and not well understood disease, HIV infection $[15,16]$.

However, tuberculosis is also one of the slowest acting infectious disease agents known, making it possible to intervene in its transmission dynamics and dramatically change both the prevalence of latent infection and the incidence of active, infective cases in a population [17]. Although tuberculosis is mostly a respiratory illness and almost always spreads through the bacteria being carried on droplets of secretions expelled via talking or coughing by persons with active infections, tuberculosis infection is unlikely to be caused by a casual contact in a single incident. It is highly dependent on prolonged, repeated, and close contact with an active case, such as within a household, within institutional settings like prisons, nursing homes, homeless shelters, group homes, and military barracks. In addition to frequency and proximity of contact, the lack of ventilation of the air and lack of exposure to ultraviolet light may increase opportunities for infection [18].

Once an active case is recognized, that person can be treated to push the infection back into latency, not a cure but effective in stopping transmission and greatly reducing both morbidity and mortality. Inadequate or incomplete treatment courses can backfire, producing drug-resistant TB strains that are much more difficult and resource intensive to treat, while also allowing a case to remain infective. The recommendation of the World Health Organization for treatment of tuberculosis disease has been with directly observed short course therapy (DOTS), where individuals are required to come into clinics to take chemotherapeutics targeted at Mycobacterium tuberculosis. Ideally, every means of reducing that window of infectivity from detection of active cases through completion of treatment courses should be employed to prevent a resurgent TB epidemic. Yet, controlling and preventing the spread of tuberculosis, HIV and other interrelated infectious diseases are just one of many critical missions that public health agencies are tasked to accomplish. In this setting, finding theoretically sound yet accessible ways to model factors important in the spread of these infections in order to target scarce public health intervention resources is critical.

In the USA and worldwide the goal for the elimination of tuberculosis has been set, with the primary strategy for accomplishing this goal, the implementation and expansion of directly observed therapy (DOTS) $[5,12,13]$. With renewed funding for tuberculosis infrastructure, new methods for examining outbreak clusters, new guidelines for disease reporting, and the emphasis on DOTS in the United States, there has been a decrease of $44 \%$ in the number of cases of TB from 1993 to 2003 [15]. Nevertheless, DOTS as an exclusive strategy for elimination of TB may not be on its own adequate for this purpose [19], and even at the current rate of decline in the United States TB would not be eliminated for more than 70 years.

The social-environmental structural factors that promote Mycobacterium tuberculosis spread and increase probabilities of exposure to an infective case and the individual factors that influence both the probabilities of developing an active case and the probabilities of case detection and treatment are not distributed homogeneously throughout any American population [20-22]. In fact, not only are these factors correlated with each other; they are also spatially clustered within neighborhoods [23]. Evidence based on analysis of neighborhood data of many sites around the world suggests that there is a higher transmission of tuberculosis within dense neighborhoods and that the infection spreads to low density ones. In a recent study on tuberculosis in Taiwan it was demonstrated that "the tuberculosis incidence in a township was significantly affected by the incidence in neighboring townships" [24]. In another excellent Brazilian study conducted to analyze the dynamics of the dissemination in the town of Olinda, "it became clear that the high density household favours the rapid transmission and manifestation of the disease" [25].

In the dense metropolitan regions of the world's rapidly urbanizing populations, housing markets are highly segmented and segregated based on wealth, income, and social status, including race and ethnicity in racialized societies such as the United States. A city's residents are constrained in their choice of neighborhoods in which they can live and segregated into neighborhoods with specific clusters of characteristics.

TB is therefore highly dependent on social context and environment factors, meaning the social structure of one's cluster of closest contacts and spatial distribution of those clusters in neighborhoods matter. Traditional transmission probability models fail in that they often assume homogeneity across populations or independence of individuals' risks. For that reason, we propose to develop a stochastic automatonbased simulation of TB epidemic transmission dynamics. We believe it may be an alternative or extension to compartmental (differential equation based) transmission models which can capture probabilistic (stochastic) transmission rates in heterogeneous population groups (compartments) and compartment mixing [24], but not the spatial structuring of a clustered social/physical environment [25]. Virtual models of TB dynamics are useful as heuristic devices that enable the testing of different structural and susceptibility scenarios that may not be feasible (and certainly not ethical to experimentally create) in the real world. In addition, they offer the opportunity to estimate the impact of different treatment or prevention scenarios as we will present in this study.

Lastly, because this spatially based simulation is mapped to the structure of a prototypical urban area, visualizing the dynamics of disease transmission and the potential consequences of policy shifts affecting disease transmission probabilities within types of neighborhoods becomes accessible to audiences with no advanced quantitative training. The images of how an epidemic spreads across a city block by block or can be stopped in its tracks could be a powerful and persuasive communication tool in the deliberative process of setting public policies and allocating urban public health resources. 


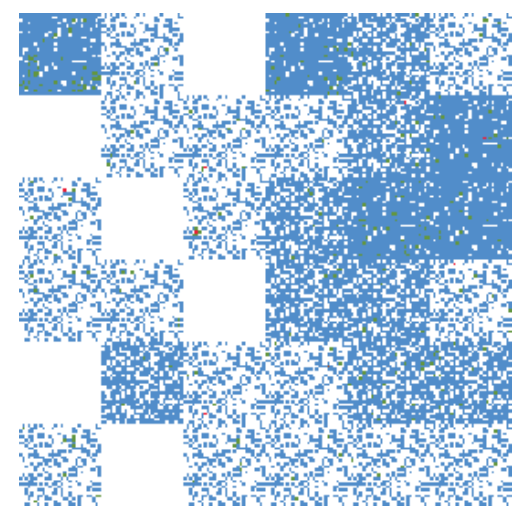

FIGURE 1: Structure and distribution of neighborhood of different densities.

\section{Methods}

2.1. Cellular Automata. Models for epidemic spread, whether stochastic or deterministic, have been based on interactions between infected populations and susceptible ones, incorporating other epidemiological features such as latency and acquisition and subsequent loss of immunity [26-30]; a similar approach was used for studying the intrinsic transmission dynamics of tuberculosis based on a multicentury analysis [31]. In all these studies susceptible individuals are assumed to mix randomly with infected ones following mass-action dynamics. This assumption has been retained even when spatial heterogeneity was considered $[3,32]$.

Because of its spatial flexibility, cellular automaton makes possible the construction of neighborhoods differing in their characteristics such as densities and vulnerabilities, thereby studying the dynamics of epidemics throughout spatially diverse populations. It is specifically appropriate for studying diseases that are mainly concentrated in deprived neighborhoods and that absorb immigrants from countries with endemic disease [33]. Thus scenarios can be simulated by placing active cases among the homeless, ex-prisoners, or immigrants in different neighborhoods and following the propagation of infection.

The stochastic automata simulation of tuberculosis transmission dynamics was implemented using J software (version $6.01)$.

2.2. Structure of the Spatial Environment: Densities and Neighborhoods. The "city" consists of 36 neighborhoods (a 6 by 6 grid), with each neighborhood defined as a grid of 3136 cells ( 56 cells by 56 cells) (Figure 1 ). The number of these cells considered to be occupied by an individual varied by the degree of crowding. Individuals are located in dense ( 0.9 probability of any cell being occupied, $n=706)$, less dense ( 0.67 probability of any cell being occupied, $n=525$ ), and least dense ( 0.33 probability of any cell being occupied, $n=259)$ neighborhoods. In addition, a few "empty" neighborhoods were included to simulate nonresidential areas within the "city."

Figure 1 shows the initial structure and distribution of neighborhoods of different densities, where blue cells indicate occupied cells $(n=12,333)$ and white cells indicate unoccupied cells. There are 5 neighborhoods of high density, 8 neighborhoods of medium density, 17 neighborhoods of low density, and 6 empty neighborhoods distributed to replicate that of a typical small city with spatial clustered neighborhoods of differing densities.

2.3. Local Interactions. The stochastic automata models presented here consist of a lattice of cells populated by individuals in one of four states: (i) noninfected, (ii) infected-latent (at various levels of progression), (iii) infected-infectious (active), and (iv) dead (Figure 2).

Each of the states is coded with numbers: 0 through 44 (Figure 3). During each time step $(t)$, a cell between states 25 and 42 ("infectious") can cause a change in a cell that is adjacent (Figure 2) or up to 4 cells away from the source.

The total initial number of latent infected individuals was 14 in high density areas (2\%), 3 in medium density areas $(0.6 \%)$, and 2 in low density areas $(0.8 \%)$.

At each level of distance from an infectious cell, other noninfected occupied cells in state 1 have the possibility of becoming infected and advancing to state 2 with probabilities shown in Figure 3 and calculated using the following equation:

$$
\begin{aligned}
P_{i}=1 & -\left(1-r w_{a}\right)^{a}\left(1-r w_{b}\right)^{b}\left(1-r w_{c}\right)^{c}\left(1-r w_{d}\right)^{d} \\
& \cdot\left(1-G r w_{e}\right),
\end{aligned}
$$

where $P_{i}=$ the probability that uninfected person will become infected; $r=$ the basic probability that an infection will be passed between two close contacts during a one month period; $a-d=$ the number of infective contacts within each of the four contact situations arranged concentrically around each cell person: ( $a$ ) the closest ring, $(b)$ the second closest, $(c)$ the third, and $(d)$ the farthest; $w_{a-d}=$ the weight or relative reduction in transmission probability that occurs with increasing distance between four classes of contacts $(0-1) ; w_{e}=$ the relative probability that an infective source will infect an uninfected person through casual contact, if all are infected; $G=$ the proportion of the total population that is infected.

Individuals in higher density clusters will in general have a higher probability of becoming infected, due to more adjacent (and possibly active) individuals.

2.4. Transition Probabilities. Figure 3 indicates the progression of stages in cells representing individuals. The progression of status of cells is as follows: occupied noninfected to latent, latent to infectious, and infectious to death, with a treatment back to step 1 possible during infectious steps 30 to 42. Within each of these states, cells may progress at different rates: 1 step per month if in the general population and 2 steps per month for immunocompromised cells, when latent or infectious. For general population infected (stages 30 to 42), probability of being treated (going back to stage 1) per cycle (month) is varied depending on the treatment scenario. Among the infected and the eligible for death (stages 25 to 42) probability of death is 0.0008372 per cycle (month). 

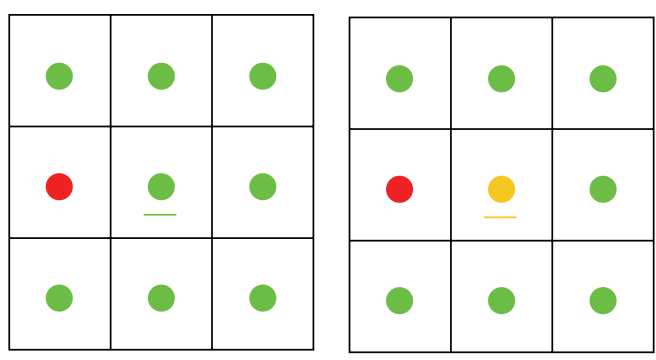

Active

Latent TB

Noninfected individual

(a) High density

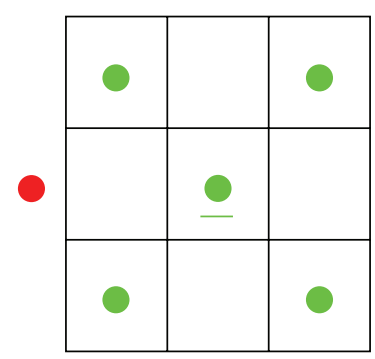

Active

Noninfected individual

(c) Low density cluster

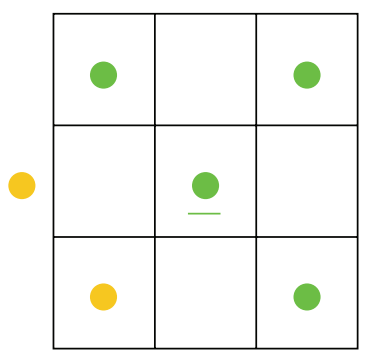

Latent TB

Noninfected individual

(d) Low density cluster

Figure 2: Example of high density (a, b) and low density (c, d) local spatial population matrixes at time 0 (a, c) and time $t$ (b, d) and effect on a particular individual (underlined dot).

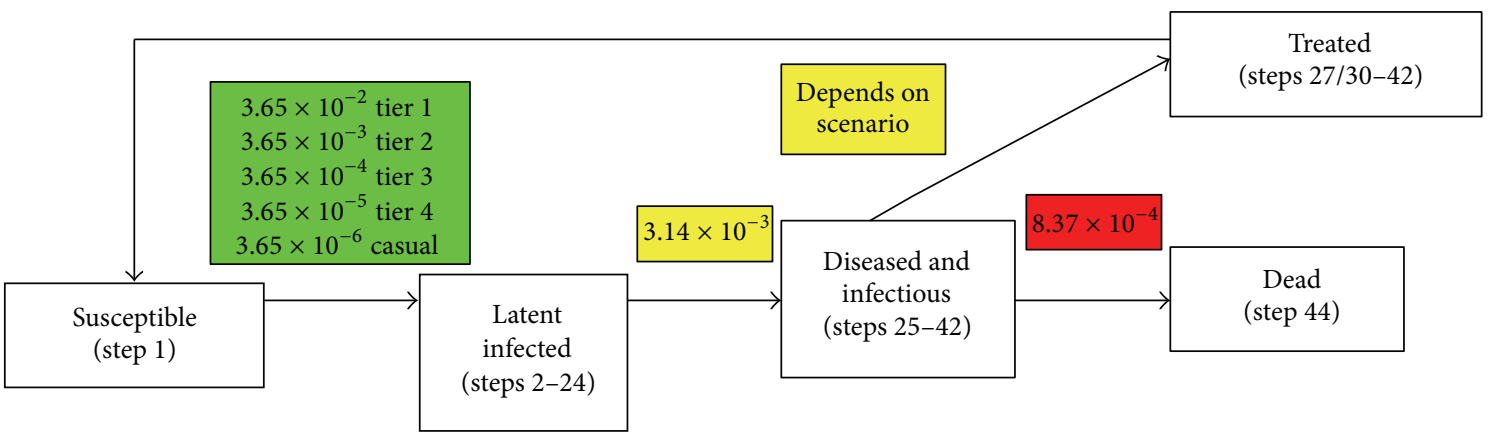

FIGURE 3: Flow diagram of states and transition probabilities per month for each individual cell.

2.5. Treatment Scenarios. The different scenarios that we evaluate with the model are based on the percent of individuals infected who receive successful treatment in the middle and low density neighborhoods and the percent of individuals who receive successful treatment in the highest density neighborhoods which are considered to be vulnerable and are referred to as "targeted treatment." We consider in each neighborhood a mix of two kinds of populations: the general infected and the immunocompromised. There is a $5 \%$ prevalence of immunocompromised individuals in the densest neighborhoods and a $1 \%$ prevalence in the medium and least dense neighborhoods. The model scenarios are presented in the table, where percent of successful treatment in the population is the product of percent of case ascertainment and percent of successful treatment.

Mean values (and standard deviations) of 50 runs for the number of deaths, the total number of infections during the duration of the simulation, and the number of people infected within high density, medium density, and low density neighborhoods (with results for treatment possible beginning 6 months after initial infection or beginning 3 months after initial infection are all presented in Table 1).

The no treatment model is presented as baseline. The model that simulates $40 \%$ successful treatment across neighborhoods is based on the level of treatment that is standard practice in many areas. $70 \%$ treatment is a level that is reasonably achieved with a high level of surveillance and funding for treatment. 90\% case ascertainment and treatment is what may be achieved through high levels of surveillance and funding for close to complete treatment.

\section{Results}

All scenarios were run 50 times. Mean values for number of infected individuals after 240 months in low density, medium density, and high density neighborhoods and the total number of individuals who are infected during the course of the simulation, including the number of deaths, are given in the table.

As shown in Table 1, 6 treatment scenarios were evaluated. A moderate level (scenario 2) of successful treatment such as when $40 \%$ of the population is treated dramatically reduced the total number of infections as compared to no treatment (scenario 1), from 574 to 56; this reduction of $90 \%$ is seen across all neighborhoods. Increasing the treatment in high density areas from $40 \%$ to $90 \%$ with a $40 \%$ coverage in the general population (scenario 3 ) results in an additional decrease of approximately $25 \%$ in the number of infected individuals overall. A similar gain is shown over scenario 2 with treating all individuals at a 70\% level (scenario 4). Further increasing the targeted treatment from $70 \%$ to $90 \%$ while keeping $70 \%$ treatment in the general population 
TABle 1: Treatment scenarios and resulting number of deaths and number of people infected by type of area after 240 months.

\begin{tabular}{|c|c|c|c|c|c|c|c|c|c|c|}
\hline \multirow{2}{*}{$\begin{array}{l}\text { Scenarios } \\
\text { Percentage of people } \\
\text { treated in each set of } \\
\text { treatments } \\
\text { Delay in diagnosis }\end{array}$} & \multicolumn{2}{|c|}{ Deaths } & \multicolumn{2}{|c|}{$\begin{array}{l}\text { Total number of } \\
\text { infected people }\end{array}$} & \multicolumn{2}{|c|}{$\begin{array}{l}\text { Number of infected } \\
\text { people in high density } \\
\text { areas }\end{array}$} & \multicolumn{2}{|c|}{$\begin{array}{l}\text { Number of infected } \\
\text { people in medium } \\
\text { density areas }\end{array}$} & \multicolumn{2}{|c|}{$\begin{array}{l}\text { Number of infected people } \\
\text { in low density areas }\end{array}$} \\
\hline & $6 \mathrm{mo}$ & $3 \mathrm{mo}$ & $6 \mathrm{mo}$ & $3 \mathrm{mo}$ & $6 \mathrm{mo}$ & $3 \mathrm{mo}$ & $6 \mathrm{mo}$ & $3 \mathrm{mo}$ & $6 \mathrm{mo}$ & $3 \mathrm{mo}$ \\
\hline $\begin{array}{l}\text { (1) } 0 \% \text { general } \\
0 \% \text { targeted }\end{array}$ & $4(3)$ & $4(2)$ & $574(170)$ & $560(146)$ & 440 (147) & 428 (138) & 109 (55) & $110(61)$ & $20(15)$ & $19(17)$ \\
\hline $\begin{array}{l}\text { (2) } 40 \% \text { general } \\
40 \% \text { targeted }\end{array}$ & $0(0)$ & $0(1)$ & $56(14)$ & $32(8)$ & $43(12)$ & $25(7)$ & $11(7)$ & $5(3)$ & $2(1)$ & $2(1)$ \\
\hline $\begin{array}{l}\text { (3) } 40 \% \text { general } \\
90 \% \text { targeted }\end{array}$ & $0(0)$ & $0(0)$ & $44(12)$ & $27(6)$ & $33(9)$ & $20(5)$ & $9(6)$ & $5(2)$ & $2(1)$ & $2(1)$ \\
\hline $\begin{array}{l}\text { (4) } 70 \% \text { general } \\
70 \% \text { targeted }\end{array}$ & $0(0)$ & $0(0)$ & $46(13)$ & $22(6)$ & $36(11)$ & $17(4)$ & $8(5)$ & $4(2)$ & $2(1)$ & $2(1)$ \\
\hline $\begin{array}{l}\text { (5) } 70 \% \text { general } \\
90 \% \text { targeted }\end{array}$ & $0(0)$ & $0(0)$ & $48(13)$ & $22(4)$ & $37(12)$ & $17(3)$ & $8(5)$ & $3(1)$ & $2(1)$ & $2(1)$ \\
\hline $\begin{array}{l}\text { (6) } 0 \% \text { general } \\
90 \% \text { targeted }\end{array}$ & $1(1)$ & $0(1)$ & $140(62)$ & $135(73)$ & $35(11)$ & $20(10)$ & $85(57)$ & $89(67)$ & $18(17)$ & $25(27)$ \\
\hline
\end{tabular}

(scenario 5) did not make a difference. With only targeted treatment of $90 \%$ among individuals (scenario 6) in the highest density neighborhoods, there is more than twice the number of infections as compared to general treatment at the $40 \%$ level (scenario 2).

In order to examine the importance of duration of time between latent infection and the possibility of treatment we also ran all treatment scenarios with possible treatment beginning at 3 months rather than 6 months. As shown in the table, this resulted in a substantial decline in the number of infected individuals in all types of regions, most significantly.

In Figure 4, the spatial distribution of occupied cells (blue) is shown, along with black dots which indicate the 19 initially infected individuals. The impact of $40 \%$ successful treatment of all cases on the spatial distribution of infected individuals can clearly be observed (scenario 2). Due to a higher likelihood of treatment of initial case clusters, cases are not focused in the areas of initial cases. Cases tend to be spread in neighborhoods adjacent to neighborhoods where initial infections occurred and to be widely distributed.

\section{Discussion}

Mathematical models, in particular those with spatial components, may be useful in situations where actual data are not available, where there is interest in investigating disease processes over long periods of time, where it is difficult to separate individual and spatial attributes within data, and where it is difficult or unethical to conduct randomized experiments. They may also be useful for informing policy on different types of treatment and prevention options for controlling infection and disease.

In examining the impact of evenly distributed case ascertainment and treatment, our results indicate that this appears insufficient in limiting the spread of TB in densely populated areas, especially among those with a number of susceptible individuals (due to, e.g., age, HIV, or diabetes). Our results support additional treatment beyond $40 \%$ of successful case ascertainment and treatment, especially treatment and control programs in dense, high risk neighborhoods, similar to findings from studies on programs targeting homeless groups $[34,35]$. Additional focused case ascertainment and treatment of $90 \%$ among densely populated areas with a higher number of susceptible individuals resulted in $25 \%$ fewer cases. Targeting treatment solely at residents in the very dense neighborhoods, even though at high levels (90\%), was less effective than treating the general population at a level of $40 \%$ (scenario 6 versus scenario 2).

All the transition probabilities are approximations from the literature. As in all models, this simulation makes simplifications of reality and is based on numerous assumptions. These assumptions should and can be tested systematically over ranges of starting conditions and settings in a series of sensitivity analyses. Refinements (including diabetes as another class of susceptible) and extensions (age structures and competing mortality risk) of the basic stochastic automata model are both possible and necessary.

Other limitations are the simplified states over transmission models that have been demonstrated to produce incidence, prevalence, and mortality rates consistent with historical data [36]. We did not include a separate state for noninfectious tuberculosis. In addition, we did not allow for direct paths between susceptible and infectious, but rather required that individuals pass through the latent infectious state initially. A more detailed state transition model is possible within the stochastic automata framework. Due to the small number of individuals that pass directly from susceptible to disease we do not expect this to have a substantive impact on our results. In addition, future analyses doing uncertainty analyses with a probability distribution of each transmission parameter in the model will be useful to validate these results and determine the extent to which our results are robust to potential errors or temporal changes in parameter estimates.

The simulation results produced some answers to the set of questions we proposed regarding different treatment 


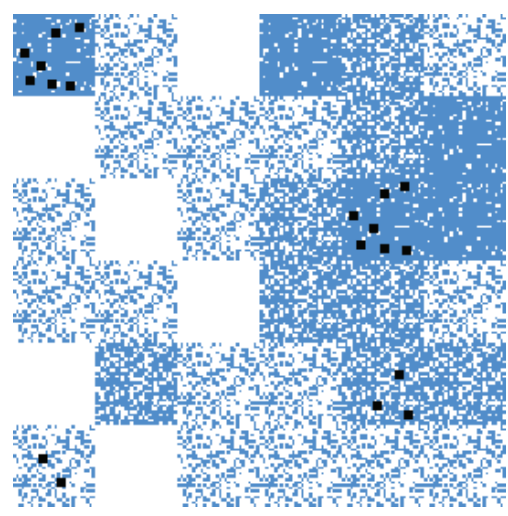

Initial distribution

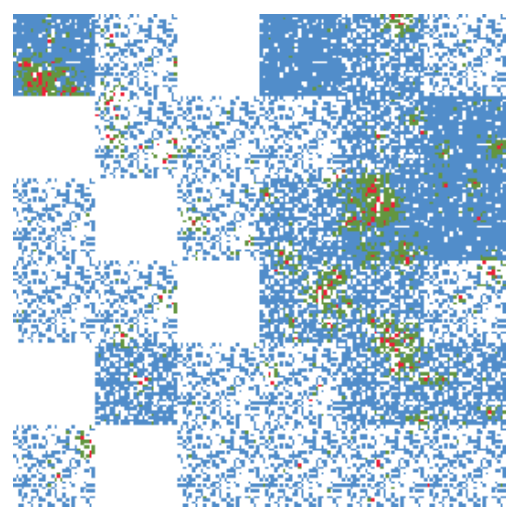

Scenario 1

(no treatment)

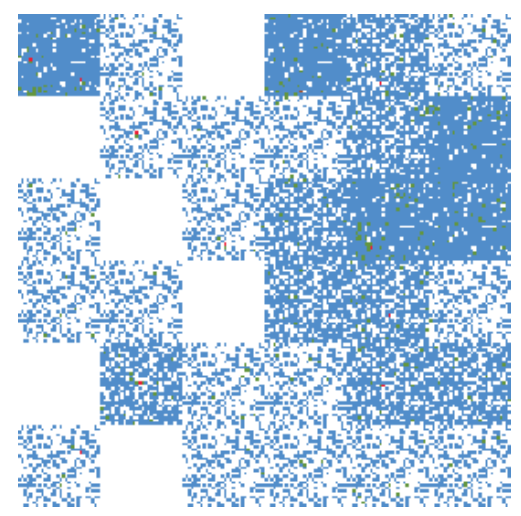

Scenario 2

$40 \%$ treatment of all

FIGURE 4: Spatial distribution of infected cases and deaths: initial (black), occupied (blue), latent (green), early stage (yellow), and late stage (red).

scenarios. Certainly, TB has an explosive spread in the dense neighborhoods and into contiguous dense neighborhoods, especially when active cases are initially seeded in those neighborhoods, and those neighborhoods also have higher proportions of more susceptible persons. Even if a dense neighborhood is somewhat isolated from areas with high levels of epidemic spread, there is still significant spread within that neighborhood. But there is also a high degree of patchiness in TB spread, with less active cases spreading in medium density neighborhoods, even if initially seeded with active cases. In isolated low density neighborhoods there is very limited spread of disease. Eventually, however, there are active cases in neighborhoods, even if not initially seeded with active cases. But all this is in the case of no effective treatment of active cases.

For some of our parameters there was not an extensive literature on which to base parameter estimates. Notably the proportion of infections with decreasing but still close proximity is not well documented, and other models of tuberculosis disease have demonstrated that number of new infections from an infectious case contribute importantly to the disease dynamics [36].

As we see cellular automata models may be useful in answering fundamental questions regarding the relationship between socioeconomic characteristics of populations and disease spread. Here it helped us identify the course of spread across segregated neighborhoods over a chosen period of time. In addition we demonstrated its value in assessing treatment strategies, such as identifying populations to be targeted by the sociodemographic makeup of their neighborhood. Important questions remain open, for example, how would housing policies affect the course of TB spread through changes in crowding and demographic characteristics? Immigration from a region where TB has been endemic and highly prevalent is a risk factor for latent infection in the USA. Immigrant populations are clustered in neighborhoods. How would this affect the spread across other neighborhoods? If individual level differences in populations have a large impact on the spread of $\mathrm{TB}$, which has the bigger impact differential susceptibilities or treatment rates? Stochastic or cellular automata have the potential of becoming a powerful tool for answering questions to guide policy makers and support their decisions.

\section{Conflict of Interests}

The authors declare that there is no conflict of interests regarding the publication of this paper.

\section{Authors' Contribution}

David H. Rehkopf, Sc.D., conceived the study with AFD, implemented the model for various treatment scenarios, and interpreted the analytical results. Alice Furumoto-Dawson, Ph.D., conceived the study with DHR and prepared the background on which the study was designed as articulated in the Introduction. In addition she provided through her research estimate values of the parameters in the model. Anthony Kiszewski, Ph.D., developed the mathematical and computational aspects of the stochastic cellular automata used in this study and supervised its implementation. Tamara Awerbuch-Friedlander, Ph.D., supervised all aspects of the study implementation, contributed to the interpretation of the results, and edited the final versions of the paper. All authors helped to develop ideas and choose scenarios for investigation and wrote parts of the paper.

\section{Acknowledgments}

The authors wish to acknowledge the contribution of the course "Mathematical Models in Biology" offered in the spring of 2003 at the Harvard School of Public Health for the interdisciplinary approach that motivated the study. The authors thank Nathalie Marchand and Isabel Madzorera for their editorial contribution. 


\section{References}

[1] T. Toffoli, "Cellular automata as an alternative to (rather than an approximation of) differential equations in modeling physics," Physica D. Nonlinear Phenomena, vol. 10, no. 1-2, pp. 117-127, 1984.

[2] A. E. Kiszewski, T. Awerbuch, S. Sandberg, and A. Spielman, "A cellular automata model for exploring the relation between sexual behaviour and fertility in ticks," Journal of Biological Systems, vol. 5, no. 2, pp. 203-213, 1997.

[3] B. Bolker and B. T. Genfell, "Space, persistence and dynamics of measles epidemics," Philosophical Transactions of the Royal Society B: Biological Sciences, vol. 348, pp. 309-332, 1995.

[4] A. E. Kiszewski and T. Awerbuch-Friedlander, "The effect of patchy host distribution on the dynamics and persistence of directly-transmitted pathogens: a cellular automata study," Applied Mathematics, vol. 4, no. 10, pp. 68-76, 2013.

[5] C. J. Rhodes and R. M. Anderson, "Epidemic thresholds and vaccination in a lattice model of disease spread," Theoretical Population Biology, vol. 52, no. 2, pp. 101-118, 1997.

[6] L. López, G. Burguerner, and L. Giovanini, "Addressing population heterogeneity and distribution in epidemics models using a cellular automata approach," BMC Research Notes, vol. 7, article 234, 2014.

[7] S. D. Webb, M. J. Keeling, and M. Boots, "A theoretical study of the role of spatial population structure in the evolution of parasite virulence," Theoretical Population Biology, vol. 84, no. 1, pp. 36-45, 2013.

[8] S. P. Ellner, "Pair approximation for lattice models with multiple interaction scales," Journal of Theoretical Biology, vol. 210, no. 4, pp. 435-447, 2001.

[9] M. Gandy and A. Zumla, "The resurgence of disease: social and historical perspectives on the 'new' tuberculosis," Social Science and Medicine, vol. 55, no. 3, pp. 385-396, 2002.

[10] Z. Santic and K. Galic, "Epidemiology of tuberculosis during the period 1703-2011: Honoring the World Tuberculosis Day," Materia Socio Medica, vol. 25, no. 4, pp. 291-294, 2013.

[11] L. E. Thorpe, K. Laserson, S. Cookson et al., "Infectious tuberculosis among newly arrived refugees in the United States," The New England Journal of Medicine, vol. 350, no. 20, pp. 21052106, 2004.

[12] K. Lönnroth, K. G. Castro, J. M. Chakaya et al., "Tuberculosis control and elimination 2010-50: cure, care, and social development," The Lancet, vol. 375, no. 9728, pp. 1814-1829, 2010.

[13] S. D. Lawn and A. I. Zumla, "Tuberculosis," The Lancet, vol. 378, no. 9785 , pp. 57-72, 2011.

[14] D. N. Wallace, "Discriminatory public policies and the New York City tuberculosis epidemic, 1975-1993," Microbes and Infection, vol. 3, no. 6, pp. 515-524, 2001.

[15] CDC. Centers for Disease Control, “Tuberculosis-United States, 1985-and the possible impact of human T-lymphotropic virus type III/lymphadenopathey-associated virus infection," Morbidity and Mortality Weekly Report, vol. 35, pp. 74-76, 1986.

[16] B. R. Bloom and C. J. L. Murray, "Tuberculosis: commentary on a reemergent killer," Science, vol. 257, no. 5073, pp. 1055-1064, 1992.

[17] B. Song, C. Castillo-Chavez, and J. Aparicio, "Tuberculosis models with fast and slow dynamics: the role of close and casual contacts," Mathematical Biosciences, vol. 180, pp. 187-205, 2002.
[18] Centers for Disease Control and Prevention (CDC), "Controlling tuberculosis in the United States: recommendations from the American Thoracic Society, CDC, and the Infectious Diseases Society of America," Morbidity and Mortality Weekly Report, vol. 54, pp. 1-84, 2005.

[19] T. F. Brewer and S. J. Heymann, "To control and beyond: moving towards eliminating the global tuberculosis threat," Journal of Epidemiology \& Community Health, vol. 58, no. 10, pp. 822-825, 2004.

[20] M. F. Cantwell, M. T. McKenna, E. McCray, and I. M. Onorato, "Tuberculosis and race/ethnicity in the united states: impact of socioeconomic status," American Journal of Respiratory and Critical Care Medicine, vol. 157, no. 4, pp. 1016-1020, 1998.

[21] D. Acevedo-Garcia, "Zip code-level risk factors for tuberculosis: neighborhood environment and residential segregation in New Jersey, 1985-1992," The American Journal of Public Health, vol. 91, no. 5, pp. 734-741, 2001.

[22] I.-C. Ng, T.-H. Wen, J.-Y. Wang, and C.-T. Fang, "Spatial dependency of tuberculosis incidence in taiwan," PLOS ONE, vol. 7, no. 11, Article ID e50740, 2012.

[23] A. Amador, W. V. de Souza, M. F. P. M. de Albuquerque et al., "A dynamic analysis of tuberculosis dissemination to improve control and surveillance," PLoS ONE, vol. 5, no. 11, Article ID e14140, 2010.

[24] B. M. Murphy, B. H. Singer, S. Anderson, and D. Kirschner, "Comparing epidemic tuberculosis in demographically distinct heterogeneous populations," Mathematical Biosciences, vol. 180, pp. 161-185, 2002.

[25] J. S. Koopman, S. E. Chick, C. P. Simon, C. S. Riolo, and G. Jacquez, "Stochastic effects on endemic infection levels of disseminating versus local contacts," Mathematical Biosciences, vol. 180, pp. 49-71, 2002.

[26] R. M. Anderson and R. M. May, Infectious Diseases of Humans: Dynamics and Control, Oxford University Press, Oxford, UK, 1991.

[27] T. E. Awerbuch, "Evolution of mathematic models of epidemics," in Disease in Evolution, W. R. Levins and A. Spielman, Eds., pp. 232-241, New York Academy of Sciences, New York, NY, USA, 1994.

[28] N. T. Bailey, The Mathematical Theory of Infectious Diseases and Its Applications, Griffin, London, UK, 1975.

[29] W. O. Kermack and A. G. McKendrick, "Contributions to the mathematical theory of epidemics," Journal of the Royal Statistical Society, vol. 115, pp. 700-721, 1927.

[30] M. Murray, O. Oxalade, and H.-H. Lin, "Modeling social, environmental and biological determinants of tuberculosis," The International Journal of Tuberculosis and Lung Disease, vol. 15, no. 6, pp. 564-570, 2011.

[31] S. M. Blower, A. R. McLean, T. C. Porco et al., "The intrinsic transmission dynamics of tuberculosis epidemics," Nature Medicine, vol. 1, no. 8, pp. 815-821, 1995.

[32] R. M. May and R. M. Anderson, "Spatial heterogeneity and the design of immunization programs," Mathematical Biosciences, vol. 72, no. 1, pp. 83-111, 1984.

[33] P. Farmer, "Social inequalities and emerging infectious diseases," Emerging Infectious Diseases, vol. 2, no. 4, pp. 259-269, 1996.

[34] P.-M. Kong, J. Tapy, P. Calixto et al., "Skin-test screening and tuberculosis transmission among the homeless," Emerging Infectious Diseases, vol. 8, no. 11, pp. 1280-1284, 2002. 
[35] M. L. Feske, L. D. Teeter, J. M. Musser, and E. A. Graviss, "Counting the homeless: a previously incalculable tuberculosis risk and its social determinants," American Journal of Public Health, vol. 103, no. 5, pp. 839-848, 2013.

[36] T. C. Porco and S. M. Blower, "Quantifying the intrinsic transmission dynamics of tuberculosis," Theoretical Population Biology, vol. 54, no. 2, pp. 117-132, 1998. 


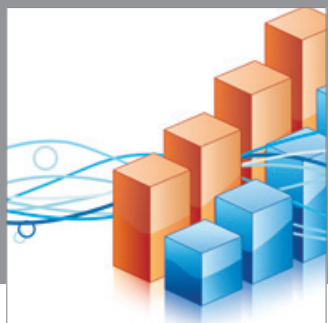

Advances in

Operations Research

mansans

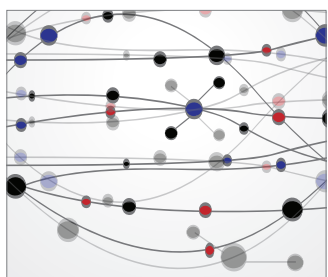

The Scientific World Journal
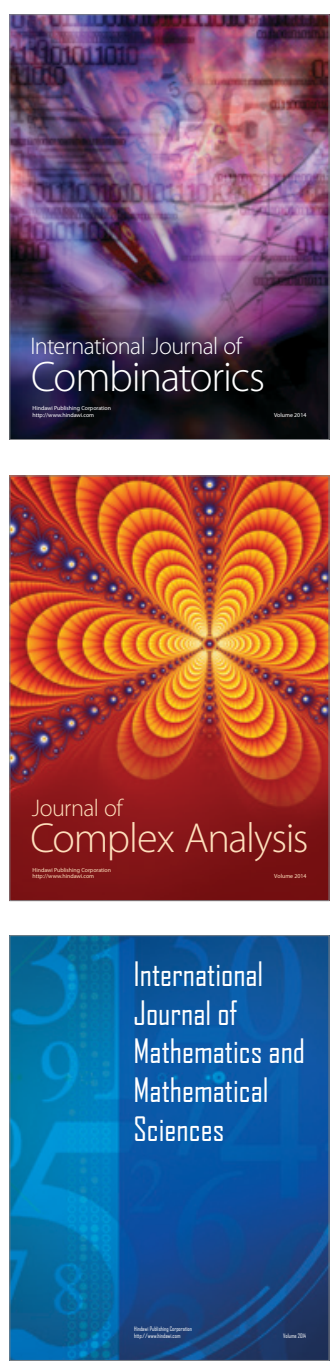
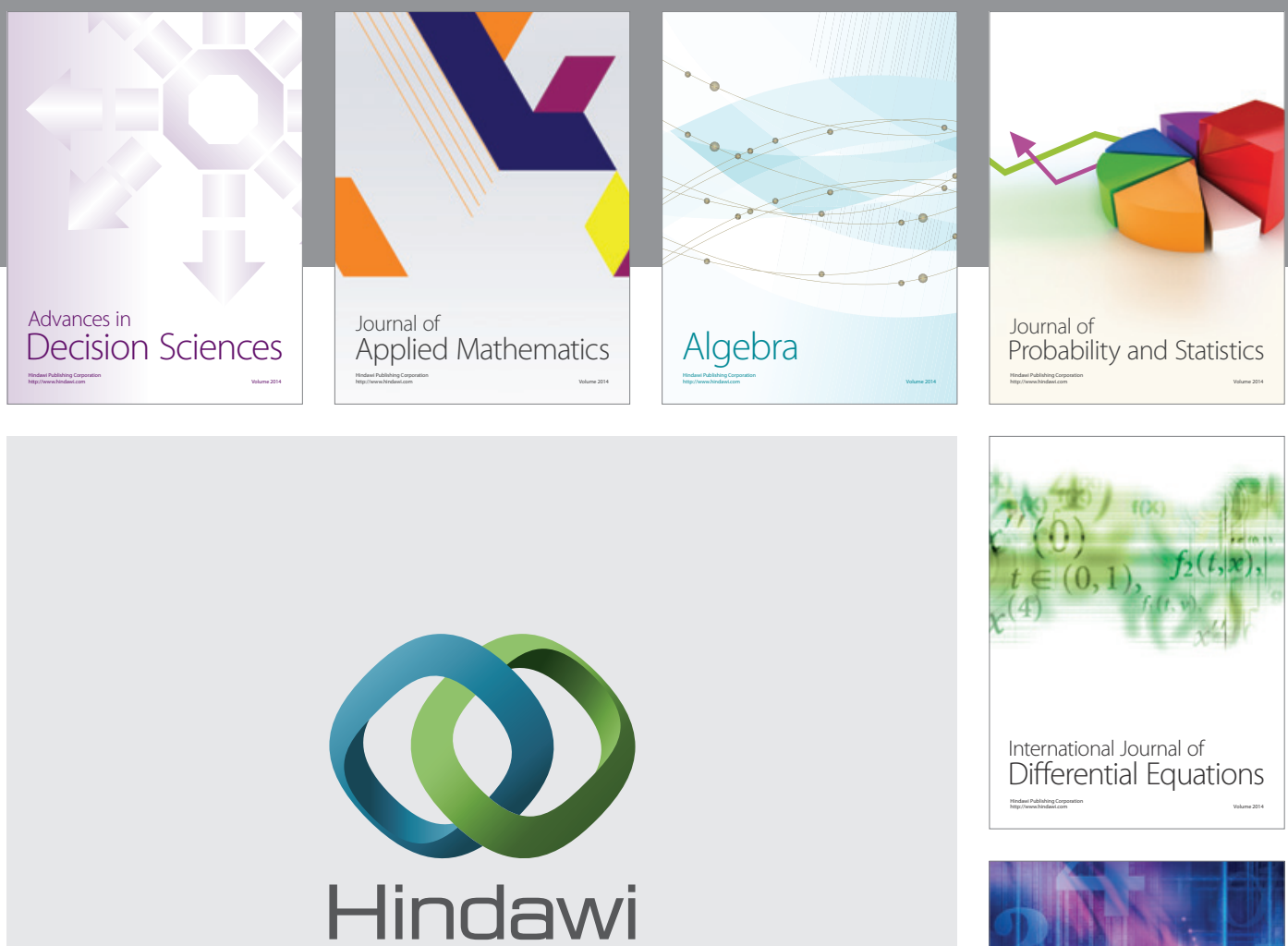

Submit your manuscripts at http://www.hindawi.com
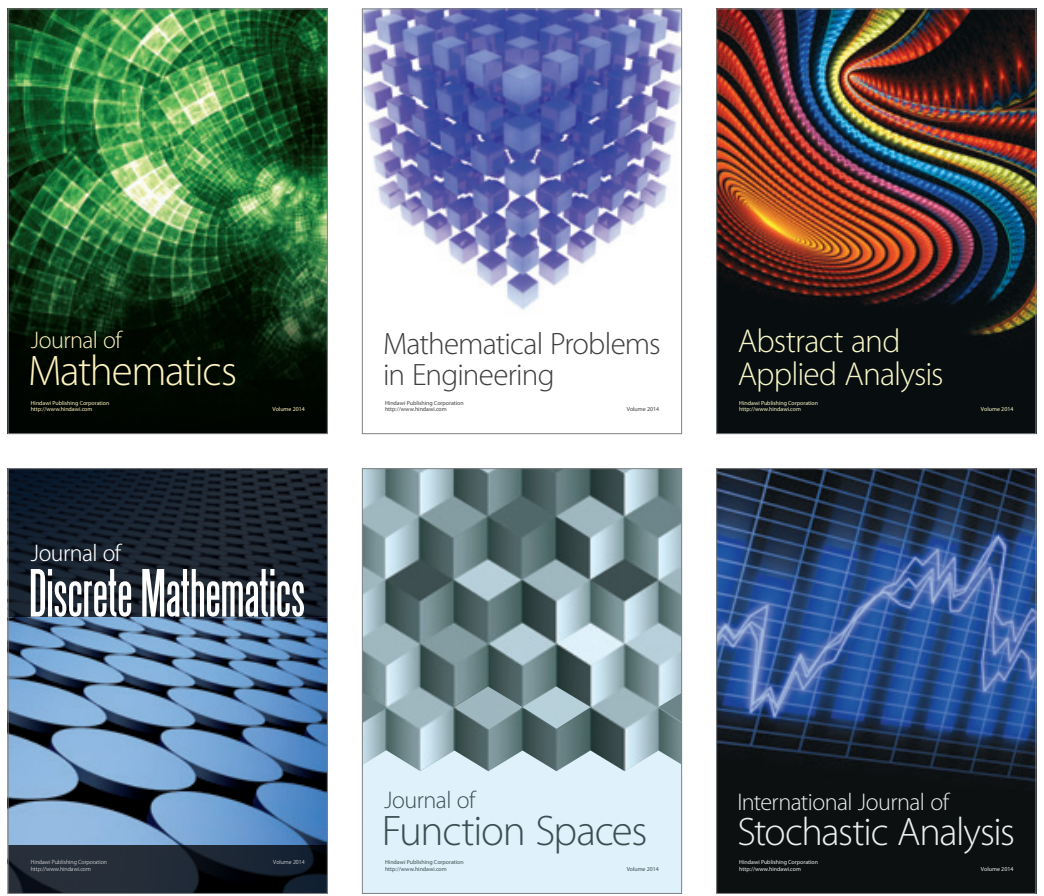

Journal of

Function Spaces

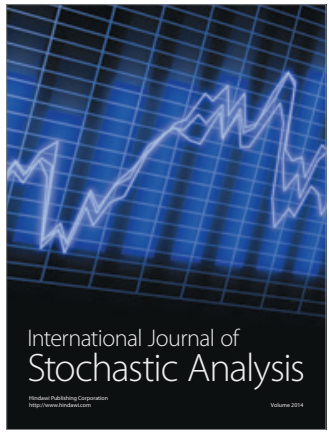

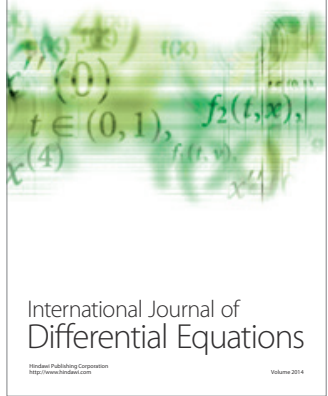
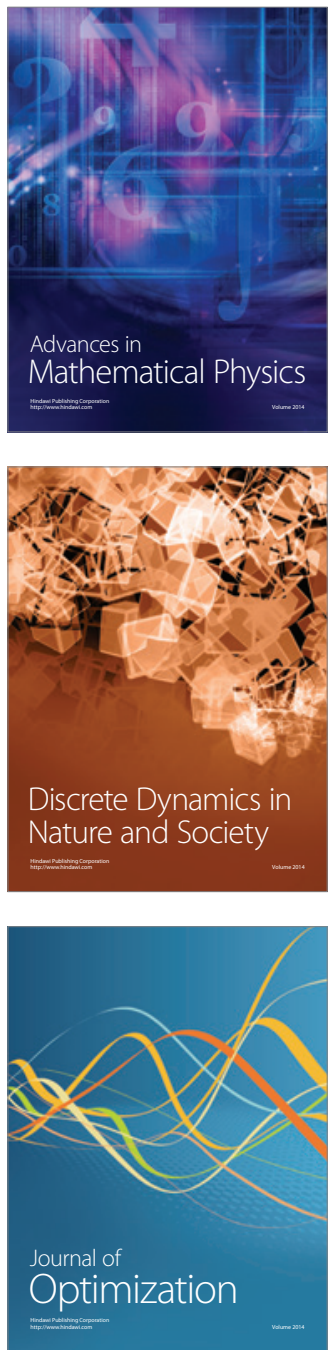